\title{
Prediction of high-speed rigid body manoeuvring in air-water-sediment
}

\author{
P. C. Chu \& G. Ray \\ Naval Ocean Analysis and Prediction Laboratory, \\ Naval Postgraduate School, Monterey, USA
}

\begin{abstract}
Falling of rigid body through air, water, and sediment with high speed is investigated experimentally and theoretically. Several experiments were conducted to shoot bomb-like rigid bodies with the density ratio similar to MK84 into the hydrographical tank. During the experiments, we carefully observe the position and orientation of the bomb-like rigid bodies. The theoretical work includes the development of 3D model for predicting high speed rigid body manoeuvring in air-water-sediment columns (STRIKE35) which contains three components: triple coordinate transform, hydrodynamics of falling rigid object in a single medium (air, water, or sediment) and in multiple media (air-water and water-sediment interfaces). The model predicts the rigid body's trajectory in the water column and burial depth and orientation in the sediment.
\end{abstract}

Keywords: body-flow interaction, bomb manoeuvring.

\section{Introduction}

Study on falling rigid body through air, water, and sediment with high water entry speed has wide scientific significance and technical application. The dynamics of a rigid body allows one to set up six nonlinear equations for the most general motion: three momentum equations and three moment-ofmomentum equations. The scientific studies of the geotechnical characteristics of a rigid body in water and sediment involve nonlinear dynamics, body and multiphase fluid interaction, body-sediment interaction, supercavitation, and instability theory.

The technical application of the hydrodynamics of a rigid body with high speed into fluid and non-fluid includes aeronautics, navigation, and civil 
engineering. Recently, the scientific problem about the movement of a rigid body in water column and sediment drew attention to the naval research. This is due to the threat of mine in the naval operations. Prediction of a fast falling rigid body in the water column contributes to the bomb strike for mine clearance in surf zones. In this study, a nonlinear dynamical system is established for the movement of a non-uniform (center of gravity not the same as the center of volume) rigid cylinder through the water-sediment interface. A bomb-strike experiment was conducted. The data collected from the experiment can be used for model development and verification.

\section{Triple coordinate systems}

Consider an axially symmetric cylinder with the centers of mass (Mc) and volume $(\mathbf{G c})$ on the main axis. Let $(L, d, \chi)$ represent the cylinder's length, diameter, and the distance between the two points (Mc, Gc). The positive $\chi$ values refer to nose-down case, i.e., the point Mc is lower than the point Gc. Three coordinate systems are used to model the falling cylinder through the air, water, and sediment phases: E-, M-, and F-coordinate systems. All the systems are three-dimensional, orthogonal, and right-handed (Chu et al. [1]).

The E-coordinate is represented by $\mathrm{F}_{\mathrm{E}}(\mathrm{O}, \mathbf{i}, \mathbf{j}, \mathbf{k})$ with the origin ' $\mathrm{O}$ ', and three axes: $x$-, $y$ - axes (horizontal) with the unit vectors $(\mathbf{i}, \mathbf{j}$ ) and $z$-axis (vertical) with the unit vector $\mathbf{k}$ (upward positive). The position of the cylinder is represented by the position of Mc,

$$
\mathbf{X}=x \mathbf{i}+y \mathbf{j}+z \mathbf{k},
$$

which is translation of the cylinder. The translation velocity is given by

$$
\frac{d \mathbf{X}}{d t}=\mathbf{V}, \quad \mathbf{V}=(u, v, w) .
$$

Let orientation of the cylinder's main-axis (pointing downward) is given by $\mathbf{i}_{\mathrm{M}}$. The unit vectors of the M-coordinate system are given by (Fig. 1)

$$
\mathbf{j}_{M}=\mathbf{k} \times \mathbf{i}_{M}, \quad \mathbf{k}_{M}=\mathbf{i}_{M} \times \mathbf{j}_{M} .
$$

Let the cylinder rotate around $\left(\mathbf{i}_{\mathrm{M}}, \mathbf{j}_{\mathrm{M}}, \mathbf{k}_{\mathrm{M}}\right)$ with angles $\left(\varphi_{1}, \varphi_{2}, \varphi_{3}\right)$ (Fig. 1). The angular velocity is calculated by

$$
\omega_{1}=\frac{d \varphi_{1}}{d t}, \omega_{2}=\frac{d \varphi_{2}}{d t}, \omega_{3}=\frac{d \varphi_{3}}{d t} .
$$

The F-coordinate is represented by $\mathrm{F}_{\mathrm{F}}\left(\mathbf{X}, \mathbf{i}_{\mathrm{F}}, \mathbf{j}_{\mathrm{F}}, \mathbf{k}_{\mathrm{F}}\right)$ with the origin $\mathbf{X}$, unit vectors $\left(\mathbf{i}_{\mathrm{F}}, \mathbf{j}_{\mathrm{F}}, \mathbf{k}_{\mathrm{F}}\right)$, and coordinates $\left(x_{\mathrm{F}}, y_{\mathrm{F}}, z_{\mathrm{F}}\right)$. Let $\mathbf{V}_{\mathrm{w}}$ be the fluid velocity. The water-to-cylinder velocity is represented by

$$
\mathbf{V}_{\mathrm{r}}=\mathbf{V}_{\mathrm{w}}-\mathbf{V}
$$

which can be decomposed into two parts,

$$
\mathbf{V}_{r}=\mathbf{V}_{1}+\mathbf{V}_{2}, \quad \mathbf{V}_{1}=\left(\mathbf{V}_{r} \cdot \mathbf{i}_{F}\right) \mathbf{i}_{F}, \quad \mathbf{V}_{2}=\mathbf{V}_{r}-\left(\mathbf{V}_{r} \cdot \mathbf{i}_{F}\right) \mathbf{i}_{F},
$$


where $\mathbf{V}_{1}$ is the component paralleling to the cylinder's main-axis (i.e., along $\mathbf{i}_{\mathrm{M}}$ ), and $\mathbf{V}_{2}$ is the component perpendicular to the cylinder's main-axial direction. The unit vectors for the F-coordinate are defined by (column vectors)

$$
\mathbf{i}_{F}=\mathbf{i}_{M}, \quad \mathbf{j}_{\mathrm{F}}=\mathbf{V}_{2} /\left|\mathbf{V}_{2}\right|, \quad \mathbf{k}_{\mathrm{F}}=\mathbf{i}_{\mathrm{F}} \times \mathbf{j}_{\mathrm{F}} .
$$
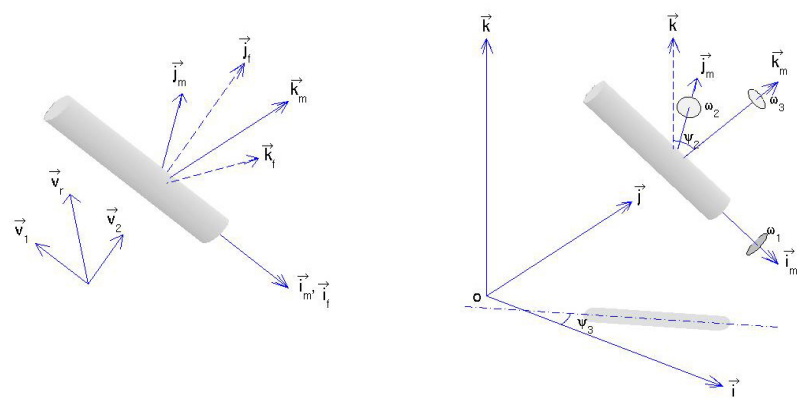

Figure 1: Three coordinate systems.

\section{Momentum balance}

The translation velocity of the rigid-body $(\mathbf{V})$ is governed by the momentum equation in the E-coordinate system (Maxey and Riley [10])

$$
\frac{d}{d t}\left[\begin{array}{c}
u \\
v \\
w
\end{array}\right]=\left[\begin{array}{c}
0 \\
0 \\
g\left(\rho_{w} / \rho-1\right)
\end{array}\right]+\frac{\rho_{w}}{\rho} \frac{D \mathbf{V}_{w}}{D t}+\frac{1}{\rho \Pi}\left(\mathbf{F}_{h}+\mathbf{F}_{V}\right),
$$

where $g$ is the gravitational acceleration; $b=\rho_{w} g / \rho$, is the buoyancy force; $\rho_{w}$ is the water density; $\Pi$ is the cylinder volume; $\rho$ is the rigid body density; $\rho \Pi=m$, is the cylinder mass; $\mathbf{V}_{w}$ is the fluid velocity in the absence of the rigid-body at the center of volume to the body. $\mathbf{F}_{h}$ is the hydrodynamic force (including drag, lift, impact forces). The drag and lift forces are calculated using the drag and lift laws with the given water-to-cylinder velocity $\left(\mathbf{V}_{r}\right)$. In the Fcoordinate, $\mathbf{V}_{r}$ is decomposed into along-cylinder $\left(\mathbf{V}_{1}\right)$ and across-cylinder $\left(\mathbf{V}_{2}\right)$ components. $\mathbf{F}_{\mathrm{V}}$ is the force caused by bubble volume variation (bubble force).

\section{Moment of momentum equation}

It is convenient to write the moment of momentum equation

$$
\mathbf{J} \cdot \frac{d \boldsymbol{\omega}}{d t}=\mathbf{M}_{w}+\mathbf{M}_{b}+\mathbf{M}_{h}+\mathbf{M}_{v},
$$

in the M-coordinate system with the body's angular velocity components $\left(\omega_{1}, \omega_{2}, \omega_{3}\right)$ defined by (4). Here, $\mathbf{M}_{\mathrm{w}}$ is the torque due to the fluid acceleration 
$\rho_{w} / \rho D \mathbf{V}_{w} / D t . \mathbf{M}_{b}$ is the torque due to buoyancy force $\mathbf{F}_{\mathrm{b}}=g\left(\rho_{w} / \rho-1\right) . \mathbf{M}_{h}$ is the hydrodynamic (drag and lift) torques. $\mathbf{M}_{\mathrm{v}}$ is the torque due to the Basset history force. In the M-coordinate system, the moment of gyration tensor for the axially symmetric cylinder is a diagonal matrix

$$
\mathbf{J}=\left[\begin{array}{ccc}
J_{1} & 0 & 0 \\
0 & J_{2} & 0 \\
0 & 0 & J_{3}
\end{array}\right],
$$

where $J_{1}, J_{2}$, and $J_{3}$ are the moments of inertia. The gravity force, passing the center of mass, doesn't induce the moment.

\section{Supercavitation}

As a high-speed rigid body penetrates into the air-water interface, an air cavity will be formed. The shape of cavity is approximately elliptical. A number of scientists have developed formulas to predict the cavity radius such as the Logvinovich [9] formula

$$
r_{c a v}=r_{\max } \sqrt{1-\frac{\left(1-r_{1}^{2} / r_{\max }^{2}\right)}{\left(1-t / t_{\mathrm{m}}\right)^{2 / \eta}}},
$$

where $r_{\max }$ is the maximum cavity radius; $t_{m}$ is the time for the formation of the cavity midpoint; $\eta(\sim 0.85)$ is the correction factor; and $r_{1}$ is the radius at location $x_{1} ; t$ is the time for the cavity formation at $x_{1}$,

$$
t=\frac{x_{M}-x_{1}}{V_{k}},
$$

where $V_{k}$ is the cavitator velocity. Recently, Dare et al. [7] proposed a simpler formula from experimental studies

$$
r_{c a v}=\frac{d}{2} \sqrt{\frac{k x_{M}}{d}+1}, \quad k=2,
$$

where $d$ is the nose diameter. The shock propagation and subsequent bubble formation may be significantly affected by the presence of an air cavity around the rigid-body. Supercavitation often occurs around the body. Cavitating flows are usually described by the cavitation number $(\sigma)$,

$$
\sigma=\frac{p-p_{v}}{\frac{1}{2} \rho_{w} V_{w}^{2}},
$$

where $p$ is the hydrostatic pressure, $p_{v}$ is the pressure in the cavity. For supercavitating flow the cavitator is located at the forward most location on the body, and the cavity downstream of the cavitator covers the body. The shape of the cavity is defined by the cavitation number. The aspect ratio (length $L$ versus diameter $d_{m}$ ) is the function of cavitation number 


$$
\lambda=\frac{L}{d_{m}}=\frac{\sigma+0.008}{\sigma(0.066+1.7 \sigma)} .
$$

Several expressions can be used for the drag coefficient. Without considering the geometry of cavity, the cavitator drag coefficient is simply calculated by (Stinebring et al. [12])

$$
C_{d}=0.82(1+\sigma) .
$$

With considering the geometry of cavity is considered, the cavitator drag coefficient is expressed by

$$
C_{d}=\left(\frac{2 r_{c a v}}{d}\right)^{2}\left(\sigma-0.132 \sigma^{8 / 7}\right)
$$

\section{Bubble dynamics}

Drag due to bubble volume variation is calculated by (Johnson and Hsieh [8])

$$
\mathbf{F}_{V}=2 \pi r_{b}^{2} \rho_{w}\left(\mathbf{V}_{w}-\mathbf{V}\right) \frac{d r_{b}}{d t},
$$

where $r_{b}$ is the bubble radius. The Rayleigh-Plesset equation (Plesset [11])

$$
r_{b} \frac{d^{2} r_{b}}{d t^{2}}+\frac{3}{2}\left(\frac{d r_{b}}{d t}\right)^{2}=\frac{1}{\rho_{w}}\left(p_{v}-p_{g} \frac{\Pi_{b 0}}{\Pi_{b}}-p-\frac{2 \tau}{r_{b}}-\frac{4 \mu}{r_{b}} \frac{d r_{b}}{d t}\right),
$$

where $p_{g}$ is the initial partial pressure of the non-condensable gas; $\Pi_{b 0}$ is the initial volume of the bubble; $\Pi_{b}$ is the volume of the bubble; $\tau$ is the surface tension; and $\mu$ is the dynamic viscosity of water.

\section{Bomb Manoeuvring Experiments (BOMEX)}

To evaluate the high-speed rigid-body manoeuvring model, scaled MK-84 and MK-65 (with and without fins) model experiments with high water entry speed were conducted during September 19-27, 2005.

\subsection{Preparation}

The overall premise of the bomb strike experiment consists of inserting various bomb-like test shapes into water, and recording their underwater trajectory over the course of the flight path. Data collection was facilitated by a pair of highspeed video cameras mounted below the water surface. Following the data collection phase of the project, all video trajectory data was converted into an array of Cartesian coordinate points which will serve as the initial data set for the development and validation of the bomb-strike prediction model (STRIKE35). 


\subsection{Bomb-like polyesters}

A collection of four bomb-like polyester resin test shapes were used. These shapes consisted of a right-cylinder, right-cylinder with hemispheric nose cone (capsule), scale model of the MK-84 GP munitions (bomb) and a modified version of the model bomb which had no stabilizing fins (shell) (Fig. 2).

The construction of the test shapes consisted of a three-part production process: prototype development, mold construction and test shape casting and finishing. This process was necessary to facilitate more efficient experimentation and to reduce the production cost of the experimental test shapes.

Prototype production began with the development of a $1 / 12$ scale replica of the real-world operational MK-84 GP bomb. This initial prototype was machined from aluminium alloy stock based on known dimensional characteristics. To create the cylinder and capsule prototypes, a polyester resin casting was created by pouring liquid plastic into a $5.08 \mathrm{~cm}$ (2") PVC mold. The shapes were allowed to cure, and then were machined to their final dimensions. As these two shapes were not intended to mimic any type of real-world munitions, their dimensions were based solely on similarity to the $1 / 12$ scale model bomb. The final prototype created was the shell shape. Construction of this design consisted of creating a polyester resin casting of the bomb prototype. The shape was then machined and sanded to remove the fins, and produce the final prototype shape. Diagrams of the final prototypes with dimensions can been seen below (Fig. 2).
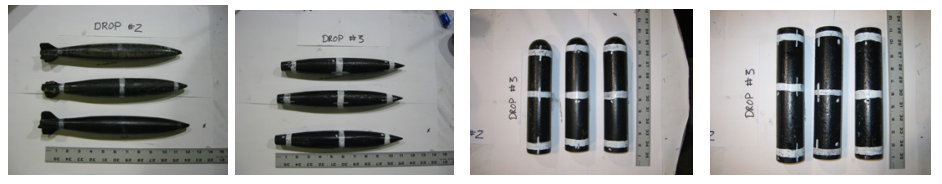

Figure 2: Various model bombs.

\subsection{Pneumatic launching device}

To facilitate the high-velocity portion of the experiment, a pneumatic launching device was created to propel the test shapes into the water at a rapid entry speed (Fig. 3). The launcher was primarily constructed of schedule 40 polyvinylchloride piping (PVC). The device consisted of three primary components: air chamber, valve mechanism, breech-load firing barrel. The air chamber was constructed of a single, five foot section of $15.24 \mathrm{~cm}(6$ ") PVC. The chamber was sealed on the end with a standard $15.24 \mathrm{~cm}$ (6") end cap, and then connected to the valve mechanism via a series of $\mathrm{PVC}$ reducer bushings. A standard $5.08 \mathrm{~cm}$ (2") PVC ball valve was fitted between the air chamber and firing barrel, and served to maintain the chamber in a pressurized state until triggered. When actuated, the valve instantaneously opened releasing the pressurized volume of air into the firing barrel. Because of the high pressures experienced by this valve, a hydraulic actuator was fitted to the device to provide the motive force necessary to open the valve. The final portion of the launcher consisted of a firing barrel. This barrel had a removable cap on the closed end to 
facilitate efficient reloading of the test shapes. Small foam sabots with fine wire lanyards which extended through a pinhole in the removable loading cap provided the means by which test shapes were held in the barrel until fired. The launcher was mounted to a steel frame oriented vertically downward with the end of the barrel positioned orthogonal to the water surface at a height of $30.50 \mathrm{~cm}$ (12") above the water. The entire apparatus was secured to the tank bridge by lag bolts and ratchet tie down straps. Additional equipment associated with launcher included a pressure indicator, emergency release valve, pneumatic fill and triggering mechanism and a 120 p.s.i. air source.
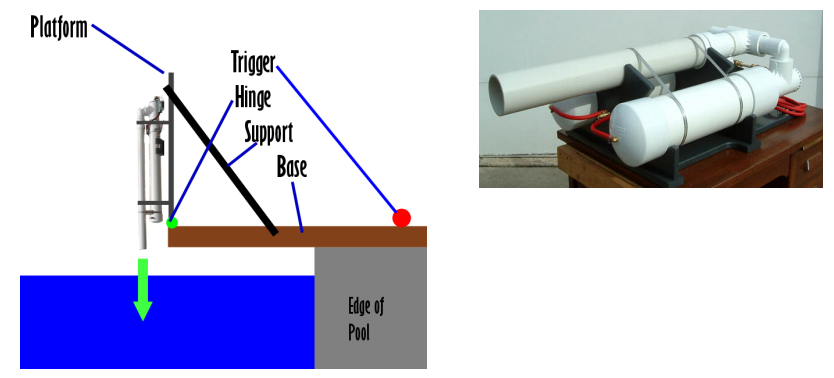

Figure 3: $\quad$ Pneumatic launching device.

\subsection{Hydrodynamic test facility}

The bomb strike experiment was conducted at the Monterey Bay Aquarium Research Institute (MBARI) Unmanned Underwater Vehicle Test Tank. This tank was used to simulate the near-shore environment frequently experienced in real-world mine countermeasure operations (Fig. 4).
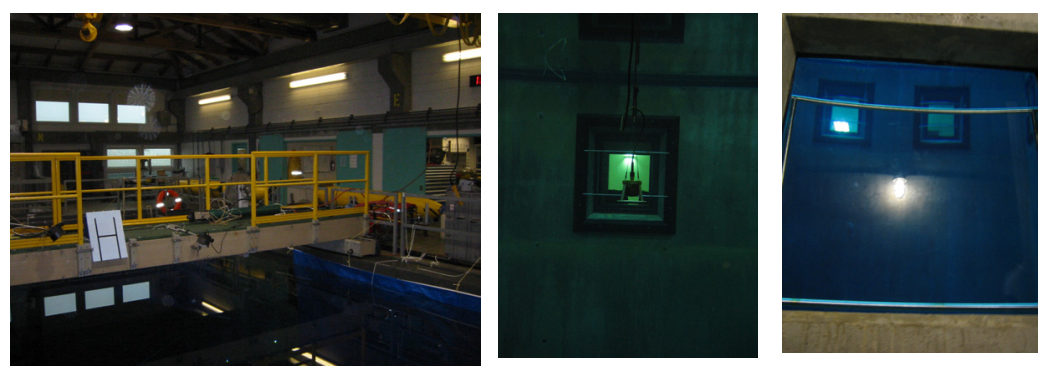

Figure 4: Hydrodynamic test facility.

The facility consists of a $9.14 \mathrm{~m} \times 13.72 \mathrm{~m} \times 9.14 \mathrm{~m}$ tank filled with standard sea water, and is contained inside a large building which provided shelter from wind and elements. A sliding bridge spans the width of the tank, and was used as a mounting surface for the pneumatic launcher and lighting equipment. Eight viewing windows located approximately six feet below the water surround the tank, and provided a venue for unimpeded sub-surface data collection to a scaled depth of roughly $36.58 \mathrm{~m}(120 \mathrm{ft})$. 
In addition to the previously mentioned equipment and facilities, a large test shape recovery device was assembled and installed in the tank prior to testing. This apparatus consisted of a $9.14 \mathrm{~m} \times 13.72 \mathrm{~m}$ net attached to a PVC gridframework constructed of $1.90 \mathrm{~cm}(3 / 4 ")$ piping. The entire apparatus was inserted horizontally across the water, and was used to recover shapes between testing runs using a series of weights and pulleys located in the corners of the tank to raise and lower the device. Lastly, two large blue tarps were placed in the tank against the walls centered in each camera's field of view.

\subsection{Motion detection equipment}

All data was collected digitally using a network of high-speed and standard video equipment and computers. Surface level information collected included experiment data and the video log. This data was collected using a pair of standard commercially available digital video camera, mounted on tripods, and located at the end of the pool directly in front of the testing zone. Both cameras operated at a $30 \mathrm{~Hz}$ frame rate. The data camera used a narrow view lens zoomed to focus on the area directly between the launcher and the water surface, and was toggled on and off between test runs. Data from this camera was later used to ascertain the initial velocity of the shapes as they entered the water. The second camera used a wide angle lens, and was employed to record a video log of the experiment. This device ran continuously throughout the experiment (Fig. 5).
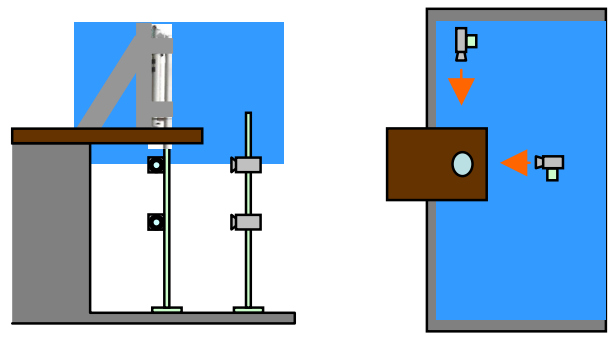

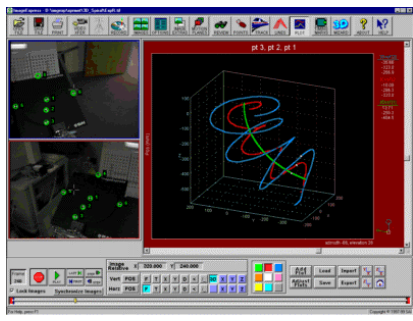

Figure 5: $\quad$ Motion detection equipment.

\subsection{Experiments}

The bomb-strike experiment consisted of a series of low velocity and highvelocity runs of the four testing shapes which were launched vertically into the water. The entry of each shape into the water was recorded by the two above surface video cameras. This above-surface data was then digitally analyzed using 3D motion analysis software to determine the initial velocity of all shapes. All below-surface data collection was facilitated by the two FASTCAM PCI high-speed cameras. The below-surface digital data was analyzed by 3D motion analysis software to determine the trajectories of each shape. All data from runs which involved malfunctions was discarded. Table 1 shows the number of test shapes which were dropped in the following sequence and repetition. 
Table 1: $\quad$ Number of experiments.

\begin{tabular}{|l|l|l|}
\hline & Low Velocity & High Velocity \\
\hline Cylinder & 8 & 12 \\
\hline Capsule & 9 & 11 \\
\hline Shell & 9 & 13 \\
\hline Bomb & 6 & 8 \\
\hline
\end{tabular}

The overall project was a two-man job conducted via handheld walkie-talkies. One man remained on or near the moveable bridge and was responsible for loading the launcher, toggling the lights and above-surface cameras and performing the launch. The other man was stationed with the high-speed cameras and computer, and served to coordinate the filming and retrieval of the below-surface data. For each individual drop, the experimenter below confirmed the readiness of the high-speed cameras and prepared the computer to save the appropriate film file. When this was confirmed, he signaled the man above, who performed the launch.
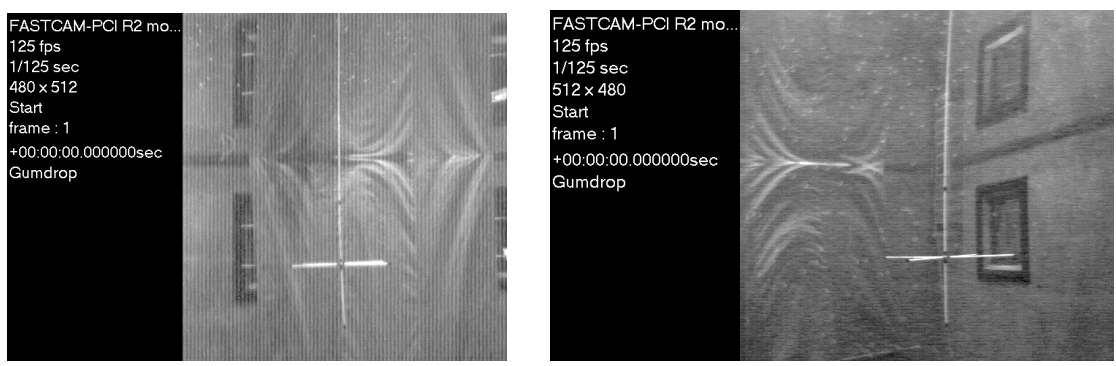

Figure 6: Trajectories of the bomb-like polyesters.

After a coordinated count conducted via the walkie-talkies, the man at the launch position fired the launcher as the man below began filming the test run. When the shape passed through the field of view of both cameras, the camera operator would cease filming, save the appropriately named file, and again signal the man above, who would then turn off the lighting and note the time and shape in the experimental record notebook. The cycle would then repeat itself until all shapes were fired. Recovery of the shapes was as described above. Digital imagery data obtained in the experiment was then analyzed to generate water trajectory data and graphics (Fig. 6).

\section{Conclusions}

(1) STRIKE35 is developed to predict high speed rigid body manoeuvring in air-water-sediment columns. It contains three components: triple coordinate transform, hydrodynamics of falling rigid object in a single medium (air, water, or sediment) and in multiple media (air-water and water-sediment interfaces). The body and buoyancy forces and their moments in the Ecoordinate system, the hydrodynamic forces (such as the drag and lift forces) 
and their moments in the F-coordinate, and the cylinder's moments of gyration in the M-coordinate. Supercavitation and bubble dynamics are also included.

(2) The momentum (moment of momentum) equation for predicting the cylinder's translation velocity (orientation) is represented in the E-coordinate (M-coordinate) system. Transformations among the three coordinate systems are used to convert the forcing terms into E-coordinate (M-coordinate) for the momentum (moment of momentum) equation.

(3) Bomb strike experiment was conducted to evaluate the 3D model.

\section{Acknowledgements}

The Office of Naval Research Breaching Technology Program (N0001405WR20209) and Naval Oceanographic Office supported this study.

\section{References}

[1] Chu, P.C., C.W. Fan, A. D. Evans, and A. Gilles, 2004: Triple coordinate transforms for prediction of falling cylinder through the water column. Journal of Applied Mechanics, 71, 292-298.

[2] Chu, P.C., A. Gilles, and C.W. Fan, 2005: Experiment of falling cylinder through the water column. Experimental and Thermal Fluid Sciences, 29, 555-568.

[3] Chu, P.C., and C.W. Fan, 2005: Pseudo-cylinder parameterization for mine impact burial prediction. Journal of Fluids Engineering, 127, 1515152.

[4] Chu, P.C., and C.W. Fan, 2006: Prediction of falling cylinder through airwater-sediment columns. Journal of Applied Fluid Mechanics, in press.

[5] Chu, P.C., C.W. Fan, A.D. Eans, A. Gilles, T.B. Smith, and V. Taber, 2006: Development and verification of 3D mine impact burial prediction model. IEEE Journal of Oceanic Engineering, in revision.

[6] Chu, P.C., 2006: Mine impact burial prediction from one to three dimensions. IEEE Journal of Oceanic Engineering, submitted.

[7] Dare, A., A. Landsberg, A. Kee, A. Wardlaw, K. Ruben, 2004: Threedimensional modeling and simulation of weapons effects for obstacle clearance. HPC Users Group Conference Proceedings, 10 pages.

[8] Johnson, V.E., and T. Hsieh, 1966: The influence of the trajectories of gas nuclei on cavitation inception. Sixth Symposium on Naval Hydrodynamics, 163-179.

[9] Logvinovich, G.V., 1969: Hydrodynamics of Flow with Free Boundaries, Naukova Dumka, Kiev, Ukraine (in Russian).

[10] Maxey, M.R., and J.J. Riley, 1983: Equation of motion for a small rigid sphere in a nonuniform flow, Phys \ics of Fluids, 26, 883-889.

[11] Plesset, M.S., 1948: Dynamics of cavitation bubbles. Journal of Applied Mechanics, 16, 228-231.

[12] Stinebring, D.R., M. L. Billet, J.W. Lindau, and R.F. Kunz, 2001: Developed cavitation-cavity dynamics, 20 pages (personal communication). 